\title{
Thermodynamical Analysis of a Two Stage Mixing Chamber R-717 Refrigeration Cycle
}

\author{
H.C. BAYRAKÇI ${ }^{a, *}$ AND S. ÇEKIN ${ }^{b}$ \\ ${ }^{a}$ Süleyman Demirel University, Department of Mechatronics Engineering, Isparta, Turkey \\ ${ }^{b}$ Süleyman Demirel University, Graduate School of Natural and Applied Sciences, \\ Department of Energy Systems Engineering, Isparta, Turkey
}

\begin{abstract}
In the process of abandoning the refrigerants which cause the greenhouse effect, R-717 has regained importance. However, energy efficiency of these refrigerant systems should be enhanced. To generalize more competitive and environmentalist systems, thermodynamic analysis of a two stage ammonia refrigeration system was performed. By using ammonia (R-717) refrigerant, energy and exergy analysis of single stage and two stage refrigeration systems were compared. In analyses EES program was used. To enhance efficiency in two stage compressions, two stage expansion and bubble through was used. Analyses were carried out with different step pressures and varied system temperatures. When the two systems were compared with each other, it was confirmed that refrigeration efficiency coefficient of two stage system is higher than that of the single stage system.
\end{abstract}

DOI: 10.12693/APhysPolA.134.21

PACS/topics: R-717, refrigeration, mixing chamber

\section{Introduction}

Protocols which are targeting to decrease emissions and which became the agenda of the refrigerating sector have become the cause for new systems design and for discussions about the refrigerant selection. The most known of these protocols are Montreal and Kyoto protocols. In this process, it was planned to put an end to usage of the refrigerants which are harmful for ozone layer. This plan was implemented. At present, regulations have been published which bring limitations of usage of ozone friendly refrigerants which have high global warming potential (GWP). European Union has brought limitations to the usage of refrigerants which have GWP value bigger than 150 (like R-134a), and was planning to gradually stop the usage of these refrigerants, according to Dang, et al. [1].

Among the alternative refrigerants, the ammonia was preferred because of its high evaporation temperature, quite low steam specific volume and the advantage of low refrigerant amount which has to be circulated through the system. Although ammonia's toxicity, substantial inflammability and explosiveness looks like disadvantages, it is used in various applications because of its perfect thermal properties. High temperatures are reached as a result of compression at single stage refrigeration processes. For this reason, abrasions can occur at compressor's components. For the purpose to prevent the temperature rise at the end of the compression process and to eliminate disadvantages of temperature rise, staged compression method was used. When the two stage compression was compared to the one stage, some advantages were found, such as compression by consuming less

*corresponding author; e-mail: cenkbayrakci@sdu.edu.tr work, lesser operating costs and bigger coefficient of performance (COP). The only disadvantage is the higher installation cost.

There are quite many reports considering these systems. Rasi [2], Czaplinksy [3], Ouadha, et al. [4], Zubair, et al. [5], Domanski [6] and Arora and Dhar [7] have studied these systems. Research for two stage compression cycles was generally performed for $\mathrm{CO}_{2}$ applications in HVAC systems by Cavallini, et al. [8], Celik [9], and for hydro fluorocarbons applications in commercial refrigeration by Torrella, et al.[10, 11], Llopis, et al. [12, 13], Ozgur and Tosun [14].

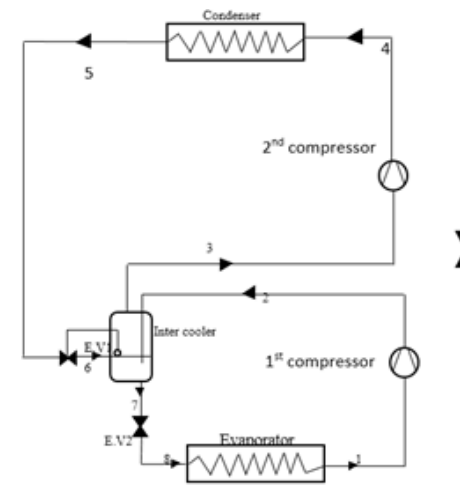

(a)

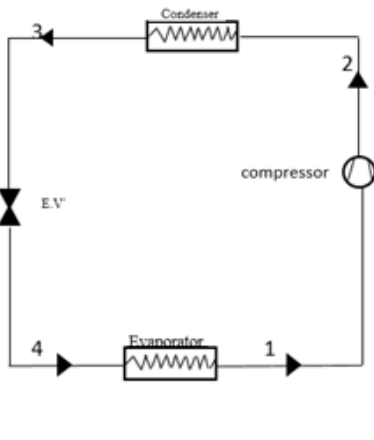

(b)
Fig. 1. Two stage (a) and single stage (b) refrigeration cycles.

\section{Theoretical analysis}

In two stage compression systems with a liquid subcooler there is a disadvantage of the cycles that the inlet gas temperature to the second compression stage may be relatively high. Especially with ammonia as refrigerant this is sometimes a problem, as was found by Granryd, et al. [15]. To solve this problem, there is an inter-cooling arrangement called "bubble through". The components of 
two stage bubble through refrigeration cycle and single stage system using R-717 are shown in Fig. 1.

In order to calculate the parameters of the refrigeration cycles some assumptions were made. These assumptions are listed below.

- For two stage system; $P_{3}=P_{2}, P_{4}=P_{5}$, $P_{6}=P_{\mathrm{INT}}=P_{7}=P_{2}$ and $s_{1}=s_{2 s}, s_{3}=s_{4 s}$ and $h_{5}=h_{6}, h_{7}=h_{8}$.

- For two stage system; $P_{\mathrm{INT}}=600 \mathrm{kPa}, T_{\mathrm{E}}=35^{\circ} \mathrm{C}$, $T_{\mathrm{c}}=50^{\circ} \mathrm{C}, Q_{\mathrm{E}}=100 \mathrm{~kW}$.

- Pressure loss in cycles was neglected.

- Heat transfers from or to the systems and components were neglected.

- Superheating value of evaporator outlet was $4{ }^{\circ} \mathrm{C}$ in the cycles.
- Efficiency value for the cycles was accepted as 0.75 .

In these assumptions, $P$ is pressure $\left(\mathrm{Nm}^{-2}\right), s$ is entropy $\left(\mathrm{JK}^{-1}\right), h$ is enthalpy $\left(\mathrm{kJ} \mathrm{kg}^{-1}\right), Q$ is heat energy $(\mathrm{J}$ or $\mathrm{kW}), T$ is temperature $\left(\mathrm{K}\right.$ or $\left.{ }^{\circ} \mathrm{C}\right)$ respectively.

Using isentropic efficiency values, enthalpy values of refrigerant at compressors' outlet obtained from the expressions are given below as Eqs. (1) and (2):

$$
\begin{aligned}
& \eta_{1 k}=\left(h_{2 s}-h_{1}\right) /\left(h_{2}-h_{1}\right), \\
& \eta_{2 k}=\left(h_{4 s}-h_{3}\right) /\left(h_{4}-h_{3}\right) .
\end{aligned}
$$

Balance equations of system components.

\begin{tabular}{l|c|c|c}
\hline \hline \multicolumn{1}{c|}{ Component } & Energy balance & Exergy balance & Mass balance \\
\hline 1st compressor & $W_{1}=m_{1}\left(h_{2} h_{1}\right)$ & $W_{1}+m_{1} e_{1}=m_{1} e_{2}+I_{\mathrm{K} 1}$ & $m_{2}=m_{1}$ \\
Inter cooler & $m_{3} h_{3}+m_{1} h_{7}=m_{3} h_{6}+m_{1} h_{2}$ & $m_{3} e_{3}+m_{1} e_{7}=m_{3} e_{6}+m_{1} e_{2}+I_{\mathrm{AS}}$ & $m_{3}=m_{6} ; m_{1}=m_{2}=m_{7}$ \\
2nd compressor & $W_{2}=m_{3}\left(h_{4} h_{3}\right)$ & $W_{2}+m_{3} e_{3}=m_{3} e_{4}+I_{\mathrm{K} 2}$ & $m_{3}=m_{4}$ \\
Expansion valve 1 & $h_{5}=h_{6}$ & $m_{6} e_{6}+I_{\mathrm{KV} 1}=m_{5} e_{5}$ & $m_{6}=m_{5}$ \\
Expansion valve 2 & $h_{7}=h_{8}$ & $m_{8} e_{8}+I_{\mathrm{KV} 2}=m_{7} e_{7}$ & $m_{7}=m_{8}$ \\
Condenser & $Q_{\mathrm{C}}=m_{3}\left(h_{4} h_{5}\right)$ & $Q_{\mathrm{C}}+m_{3} e_{5}=m_{3} e_{4}+I_{\mathrm{C}}$ & $m_{4}=m_{5}$ \\
Evaporator & $Q_{\mathrm{E}}=m_{1}\left(h_{1} h_{8}\right)$ & $Q_{\mathrm{E}}+m_{1} e_{8}=m_{1} e_{1}+I_{\mathrm{E}}$ & $m_{1}=m_{8}$
\end{tabular}

Using the balance equations given in Table I, compressor work, heat rejected from condenser, and the coefficient of performance for cooling $(\mathrm{COPc})$ we calculated. COPc for the cycles were obtained from Eq. (3):

$$
\mathrm{COP}_{\mathrm{c}}=\frac{Q_{\mathrm{E}}}{W_{1}+W_{2}} \text {. }
$$

For the calculation of two stage "bubble through" refrigerating cycle by using R-717 (ammonia), EES software was used [16]. In our notation $\eta$ represents efficiency, 2 represents second (law), "c" stands for cooling, "C" stands for condenser, "E" for evaporator, "h" for heating and "INT" stands for intercooler in the subscripts.

\section{Results}

Obtained results are presented graphically in Figs. 2-6. If the results are examined it is seen that $\eta$ and COP values of two stage cycles are better than those of single stage cycles.

In Fig. 2, $\eta_{2}$ decreases more rapidly with increasing condensing temperature than that of the two stage cycle. The highest $\eta_{2}$ was obtained between 30 and $40^{\circ} \mathrm{C}$ for both systems. Figure 3 shows COP variation with the condenser temperature. $\mathrm{COPc}$ value decreases with increasing condensing temperature. Figure 4 shows COP variations with respect to evaporator temperature. $\mathrm{COPc}$ value increases with increasing evaporation temperature. It is clearly seen that two stage compression cycle is more advantageous among the considered two cycles.

Figure 5 shows $\eta_{2}$ as function of evaporator temperature. $\eta_{2}$ increases with increasing evaporation temper-

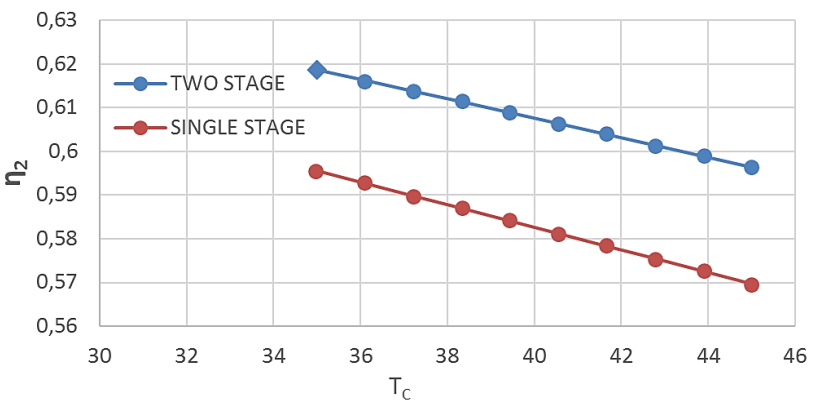

Fig. 2. Efficiency as function of condenser temperature.

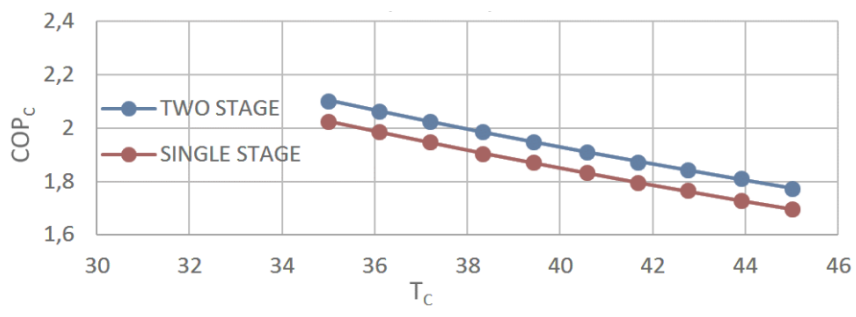

Fig. 3. COP variation with the condenser temperature.

ature. Figure 6 shows $\mathrm{COPc}$ variations with the interstage pressure at various evaporation temperatures, while condensing temperature is kept at $50^{\circ} \mathrm{C}$ for two stages cycle. For finding the change of $\mathrm{COPc}$, the inter-stage pressure was varied in the range of $400-1600 \mathrm{kPa}$. It is seen that there is an optimum value range between 600 $800 \mathrm{kPa}$. This value could be important for these kinds of systems which have large refrigeration capacities. These 


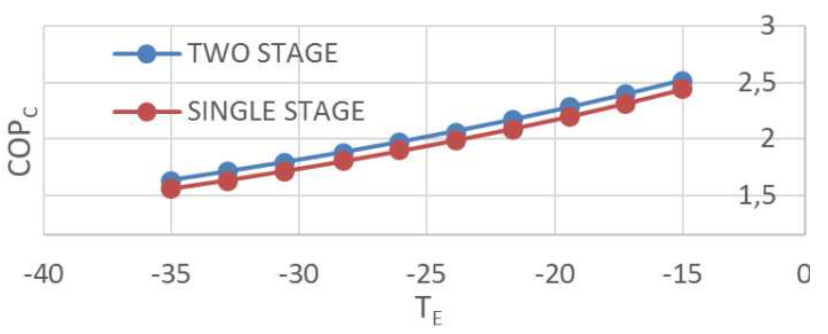

Fig. 4. COP variation with the evaporator temperature.

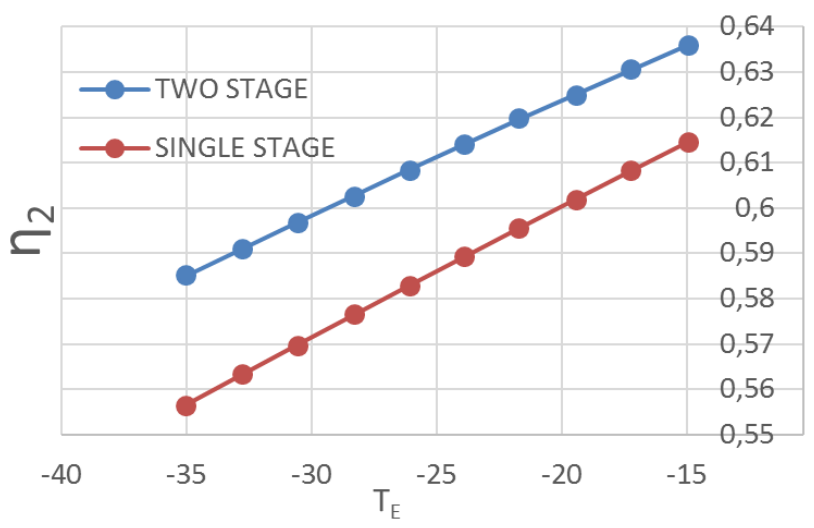

Fig. 5. Efficiency as function of evaporator temperature.

values could be considered for the selection of inter-stage pressure.

\section{Conclusions}

A comparative study was presented for two different cycles of ammonia. It was found that with the increasing evaporation temperatures $\mathrm{COPc}$ values are also increasing, however exergy efficiency values were decreasing with the condensing temperature. For lower condensing temperatures exergy efficiency reaches higher values for both cycles. Furthermore, there was an optimum value range. It was clearly seen that, "bubble through" two stage compression cycle is advantageous compared to single stage cycle. Such a comparison of energetic and exergetic performance for two stage "bubble through" R-717 refrigeration cycle gives valuable and practical knowledge for designers from the refrigeration sector.

\section{References}

[1] C. Dang, N. Haraguchi, T. Yamada, E. Hihara, Effect of Lubricating Oil on Boiling Heat Transfer of Carbon Dioxide in: 7th IIR Gustav Lorentzen Conference on Natural Working Fluids, Trondheim, Norway 2006.

[2] A. Rasi, La pression intermédiare la plus correcte pour les cycles frigorifiques $r$ deux phases in: Proceedings of the 9th International Congress of Refrigeration, Paris, France 1955.

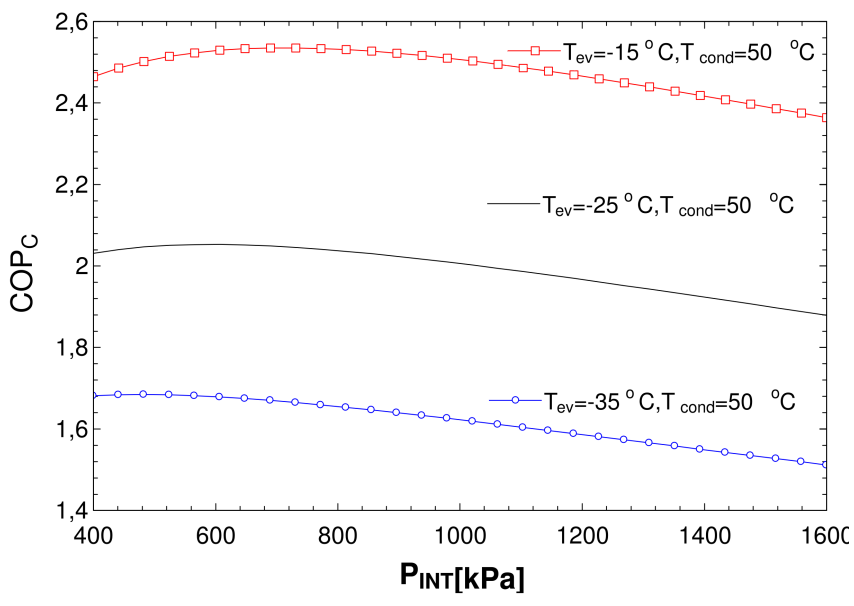

Fig. 6. COPc variation as function of inter-stage pressure for two stage cycle.

[3] S. Czaplinsky, Allgemeine Warmetechnik 91(9), 3 (1959).

[4] A. Ouadha, M. En-Nacer, L. Adjlout, O. Imine,, International Journal of Exergy 2, 14 (2005).

[5] S.M. Zubair, M. Yaqub, S.H. Khan, International Journal of Refrigeration 19, 506 (1996).

[6] P.A. Domanski, Theoretical evaluation of the vapor compression cycle with a liquid-line/suction-line heat heat exchanger, economizer, and ejector in: National Institute of Science and Technology, Interagency Report 5606, National Institute of Standards and Technology, Gaithersburg, 1995.

[7] C.P. Arora and P.L. Dhar, Optimization of multistage refrigerant compressors in: Proceedings of the 13th International Congress of Refrigeration, Paris, France 1971.

[8] A. Cavallini, L. Cecchinato, M. Corradi, E. Fornasieri, C. Zilio, International Journal of Refrigeration 28, $1274(2005)$.

[9] A. Celik, Ph.D. Thesis, Performance of two-stage $\mathrm{CO}_{2}$ refrigeration cycles, College Park: Faculty of the Graduate School of the University of Maryland 2004.

[10] E. Torrella, R. Llopis, R. Cabello, International Journal of Refrigeration 32, 307 (2009).

[11] E. Torrella, R. Llopis, R. Cabello, D. Sanchez, HVACER Research 15, 583 (2009).

[12] R. Llopis, E. Torrella, R. Cabello, J.A. Larumbe, HVACERR Research 13, 819 (2007).

[13] R. Llopis, E. Torrella, R. Cabello, D. Sánchez, Applied Energy 87, 1546 (2010).

[14] A.E. Ozgur, C. Tosun, The International Journal of Exergy 16, 127 (2015).

[15] E. Granryd, I. Ekroth, P. Lundqvist, A. Melinder, B. Palm, P. Rohlin, Refrigerating Engineering 1, 438 (2011).

[16] S.A. Klein, Engineering Equation Solver, Version 10.290-3D. F-Chart Software 2017. 